\title{
Atmospheric turbulence in phase-referenced and wide-field interferometric images
}

\section{Application to the Square Kilometre Array}

\author{
I. Martí-Vidal ${ }^{1,2}$, J. C. Guirado ${ }^{2}$, S. Jiménez-Monferrer ${ }^{2}$, and J. M. Marcaide ${ }^{2}$ \\ 1 Max-Planck-Institut für Radiastronomie, Auf dem Hügel 69, 53121 Bonn, Germany \\ e-mail: imartiv@mpifr.de \\ 2 Dpt. Astronomia i Astrofísica, Universitat de València, Dr. Moliner 50, 46100 Burjassot, Spain
}

Received 3 December 2009 / Accepted 2 April 2010

\begin{abstract}
Phase referencing is a standard calibration procedure in radio interferometry. It allows us to detect weak sources by using quasisimultaneous observations of closeby sources acting as calibrators. However, atmospheric turbulence may introduce strong differences in the optical paths of the signals of the target and calibrator and affect, or even waste, phase referencing in cases of relatively large calibrator-to-target separations and/or bad weather. The situation is similar in wide-field interferometric observations. We present the results of a Monte Carlo study of the astrometric precision and sensitivity of an interferometric array (a realization of the Square Kilometre Array, SKA) in phase-referenced and wide-field observations. These simulations can be extrapolated to other arrays by applying the corresponding corrections. We consider several effects from the turbulent atmosphere (i.e., ionosphere and wet component of the troposphere) and also from the antenna receivers. We study the changes in dynamic range and astrometric precision as a function of observing frequency, source separation, and strength of the turbulence. We find that, for frequencies between 1 and $10 \mathrm{GHz}$, it is possible to obtain images with high fidelity, although the atmosphere strongly limits the sensitivity of the instrument compared to the case with no atmosphere. Outside this frequency window, the dynamic range of the images and the accuracy of the source positions decrease. We also find that, even if a good model of the atmospheric turbulence (with an accuracy of 99\%) is used in the imaging, residual effects from the turbulence can still limit the dynamic ranges of deep, high-contrast $\left(10^{5}-10^{6}\right)$, images.
\end{abstract}

Key words. atmospheric effects - techniques: high angular resolution - techniques: interferometric - telescopes

\section{Introduction}

It is well-known that ground-based astronomical observations are affected by the atmosphere. Changes in the atmospheric opacity produce a bias in the source flux density, while changes in the refraction index distort the shape of the electromagnetic frontwave of the source. This distortion translates into a deformation of the observed source structure and/or a variation in the relative positions of all sources observed in a given field. For astronomical devices based on interferometry, atmospheric effects can be accurately modelled if the atmosphere above each element of the interferometer (hereafter, station) remains unchanged over the whole portion of the sky being observed. In these cases, the observed visibilities can be calibrated using station-based algorithms, which are relatively simple and computationally inexpensive (e.g. Readhead \& Wilkinson 1978).

However, when the spatial variations in the atmosphere are significant within the observed portion of the sky, as happens if there is atmospheric turbulence, the opacity and dispersive effects cannot be modelled as a single time-dependent stationbased complex gain over the field of view. Unless more complicated calibration algorithms are used (e.g., van der Tol 2007), the effects of these errors on the image are difficult to correct. In this paper, we study the effects that a turbulent atmosphere may introduce in interferometric observations. We focus our study on the effects produced by turbulence in the dynamic range and astrometric accuracy after a phase-referenced calibration between a strong (calibrator) source and a weak source, located a few degrees away. This study is numerically equivalent to the study of the deformation of a wide-field interferometric image at any point located at a given distance from the center of the field (i.e., the phase center of the image). In both cases, the phases introduced by the atmosphere in the signal of each antenna for the different pointing directions are the same, so the effects of the atmosphere in Fourier space (and therefore on the sky plane) will also be the same.

The results reported here are an extended version of those previously reported in the SKA memo by Martí-Vidal et al. (2009). In the next section, we describe the details of the array distribution used, as well as the characteristics of the simulated observations. In Sect. 3, we describe how the noise from the atmosphere and the receivers was added to the visibilities and in Sect. 4 describe the procedures followed in our Monte Carlo analysis. In Sect. 5, we present the main results obtained; in Sect. 6, we summarize our conclusions.

\section{Array geometry and sensitivity}

We simulated an interferometric array similar to the planned station distribution of the Square Kilometre Array (SKA). We simulated a total of 200 stations distributed in the following way: $50 \%$ are randomly distributed within a circle of $5 \mathrm{~km}$ radius (inner core); $25 \%$ are distributed outside this circle out to a 


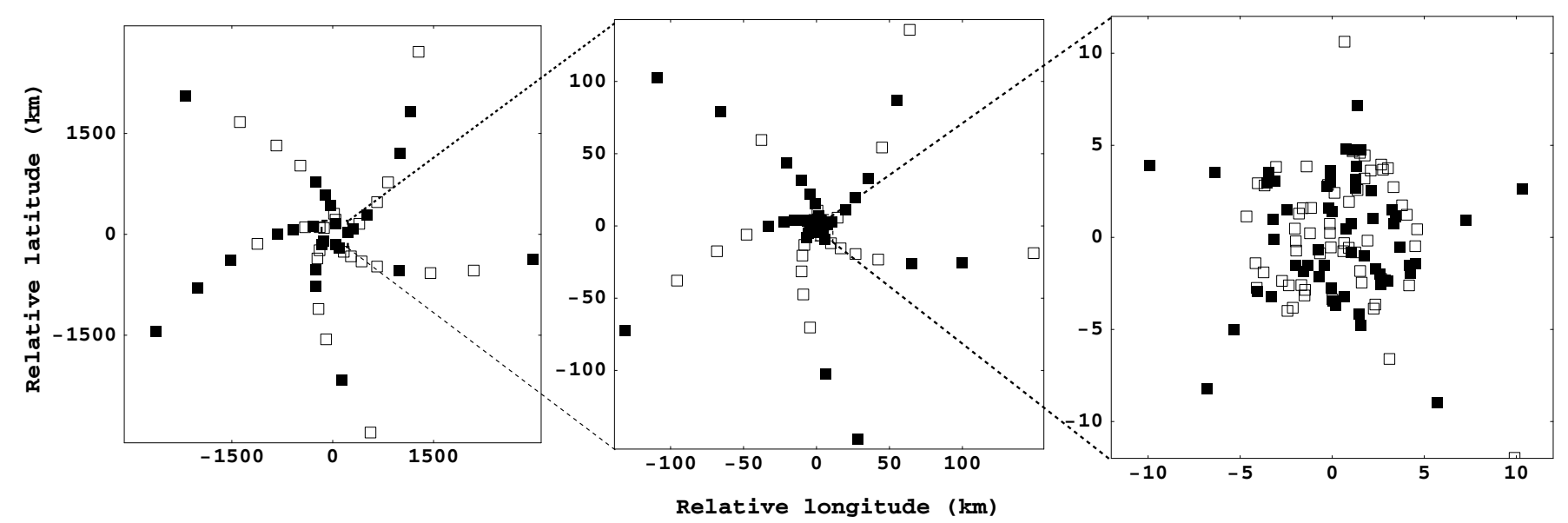

Fig. 1. Array distribution used in our simulations (empty and filled squares). Axes are relative longitude (horizontal axis) and latitude (vertical axis) in km. Left, the whole array. Center, a zoom into the core. Right, a zoom into the inner core. The stations marked with empty squares were removed from the array in a second run of our simulations, to check the dependence of the results on different array distributions (see text).

distance of $150 \mathrm{~km}$ (core), following 5 equiangular spiral arms; the remaining antennae are distributed following the same spiral arms, but out to a distance of $3000 \mathrm{~km}$ from the inner core. This array distribution is similar to that used in Vir Lal, Lobanov \& Jiménez-Monferrer (2009). The curvature of the Earth surface was taken into account in our simulations. We show the resulting array distribution in Fig. 1. We also repeated all the simulations reported here, but after subtracting a subset of 100 (randomly selected) stations from the array, to check the sensitivity of the main conclusions of this paper to different array distributions (see Appendix A). In Fig. 1, we also show the modified array after subtracting the 100 stations.

\subsection{Sensitivity and bandwidth}

We simulated interferometric observations using 16 different frequencies, spanning logarithmic bins from $150 \mathrm{MHz}$ to $24 \mathrm{GHz}$. This is the theoretical frequency window of the SKA. According to Jones (2004), the maximum observing bandwidth of the SKA will be around $25 \%$ of the central observing frequency (up to a maximum bandwidth of $4 \mathrm{GHz}$ for all frequencies above $16 \mathrm{GHz}$ ). This (maximum) frequency-dependent bandwidth translates in our simulations into a changing sensitivity of the SKA as a function of frequency.

The sensitivities of the simulated stations were also chosen to be similar to those of the SKA, which were taken from Jones (2004). These values are set for an elevation of 45 degrees and differ from those given in Schilizzi et al. (2007), but the use of the values given in Schilizzi et al. (2007), instead, does not affect the main conclusions of this paper. We interpolated the sensitivities given in Table 1 of Jones (2004) to the frequencies used in our simulations. In Fig. 2, we show the station sensitivities used.

\subsection{Source position}

We set the target source coordinates to be those of the zenith of the array center and the calibrator to an hour angle of 0 degrees, also with respect to the array center. This positioning of the sources minimizes the optical paths of the signals through the atmosphere (since both, source and calibrator, are at maximum elevations), thus enhancing the quality of the phase-referenced observations. The results given in this paper should be interpreted by taking this into account.

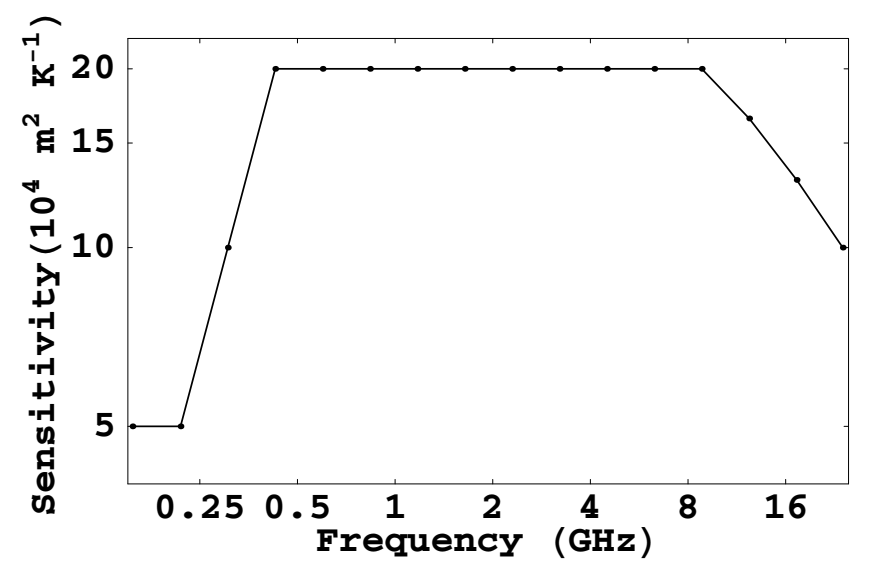

Fig. 2. Station sensitivities (i.e., effective areas over system temperatures) used in our simulations.

If the source were located far from the zenith, the mapping function of the tropospheric delay and the finite width of the ionosphere would increase the effect of turbulence on the phasereferenced visibilities of the target. In addition, the uv coverage of the interferometer would have shorter projected baselines in declination, thus decreasing the synthesized resolution in declination. Therefore, placing the sources at maximum elevations is a key limiting factor of the simulations reported here, especially if they are to be compared to real observations.

\section{Noise model}

We simulated phase-referencing observations in the following way: we assumed that the calibrator source is sufficiently strong to allow for a perfect antenna-gain calibration at its location; we then determined the image of the target source by computing the differential antenna-gain errors expected at the target location. Therefore, under the effect of atmospheric turbulence, these results depend on the calibrator-to-target separation.

We implemented two kinds of atmospheric turbulence. The first turbulence was associated with the ionosphere (the free electron content, which introduces dispersion in the radiation) and the other turbulence was associated with the wet troposphere (the water vapour, close to the Earth surface, which is not in a state of thermodynamic equilibrium). The effect of ionospheric 
turbulence on the signal phase varies as $v^{-1}$, affecting the lowfrequency observations; the effect of the wet troposphere on the phase varies as $v$, affecting the high-frequency observations. The dry troposphere (which is more homogeneously distributed over each station than the wet troposphere) was not considered in our simulations, since it can be easily modelled and removed from the data to a level lower than the effects coming from the water vapour and the ionosphere. Models of the turbulence from the ionosphere and troposphere can be found in many publications (e.g., Thomson et al. 1991). Here, suffices to say that this turbulence follows a Kolmogorov distribution. This distribution has a phase structure function given by

$D_{\phi}(\theta)=\left\langle\left(\Phi\left(\theta_{0}\right)-\Phi\left(\theta_{0}+\theta\right)\right)^{2}\right\rangle \propto \theta^{5 / 3}$,

where $\Phi\left(\theta_{0}\right)$ is the phase added by the turbulent screen to the signal of a source located at $\theta_{0}$. The brackets $<\ldots>$ represent averaging over all pointing directions located at a distance $\theta$ from the point at $\theta_{0}$. The Kolmogorov distribution is fractal-like, so both the ionosphere and wet troposphere have essentially the same phase distribution, despite a global scaling factor between them.

The global factors for both distributions (ionosphere and troposphere) were computed according to the typical values of ionospheric and tropospheric conditions. For the ionosphere, the Fried length (i.e., distance in the ionosphere for which the structure function rises to $1 \mathrm{rad}^{2}$ ) was set to be $3 \mathrm{~km}$ at $100 \mathrm{MHz}$. For the wet troposphere, we set the parameter $C_{n}^{2} L$ (i.e., the integral of the profile of $C_{n}^{2}$ along the zenith direction) to be $10^{-11} \mathrm{~m}^{1 / 3}$ (see Eq. (13.100) and Table 13.2 of Thomson et al. 1991); this value translates into a Fried length of $3 \mathrm{~km}$ for a frequency of $\sim 22 \mathrm{GHz}$. Since the Kolmogorov distribution is self-similar, it is possible to adapt the results here reported to any other atmospheric conditions (see Sect. 5.3), by scaling accordingly the source separation to the Fried length of the ionosphere (for low-frequency observations) or the wet troposphere (for high-frequency observations). We note that the selfsimilarity of the tropospheric turbulence does not apply on very large scales (the typical baseline lengths in VLBI observations), since there is a saturation in the power spectrum of the distribution (see, e.g., Thomson et al. 1991). However, this is not important to our analysis, since we did not use the absolute phase of the signal coming from a given direction in the sky, but computed the differential effects at each station from two different (closeby) directions, which depend on short-scale turbulence. Therefore, the saturation of tropospheric turbulence on large scales does not affect our results.

We computed the differential effects from the turbulent atmosphere in two ways. For the antennas of the core (within the central $300 \mathrm{~km}$ ), we generated synthetic phase screens for the ionosphere and troposphere. We show an example of one such screen in Fig. 3. We notice that this figure could represent either ionospheric or tropospheric turbulence in our modelling, just by scaling the screen by the corresponding factor. Two different screens were generated in each Monte Carlo simulation. The screen for simulating the ionosphere was placed at at a height of $300 \mathrm{~km}$ and the screen for simulating the troposphere at a height of $5 \mathrm{~km}$. For the antennas out of the core, we computed the term $\Phi\left(\theta_{0}\right)-\Phi\left(\theta_{0}+\theta\right)$ separately. We proceeded in this way (i.e., we generated a phase screen only for the core antennas, thus without generating a much larger screen for the whole array), because the distances between stations outside the core are large enough to ensure that the cross-correlation of turbulence above different stations is negligible compared to the correlation between turbulence on the calibrator and turbulence on the target

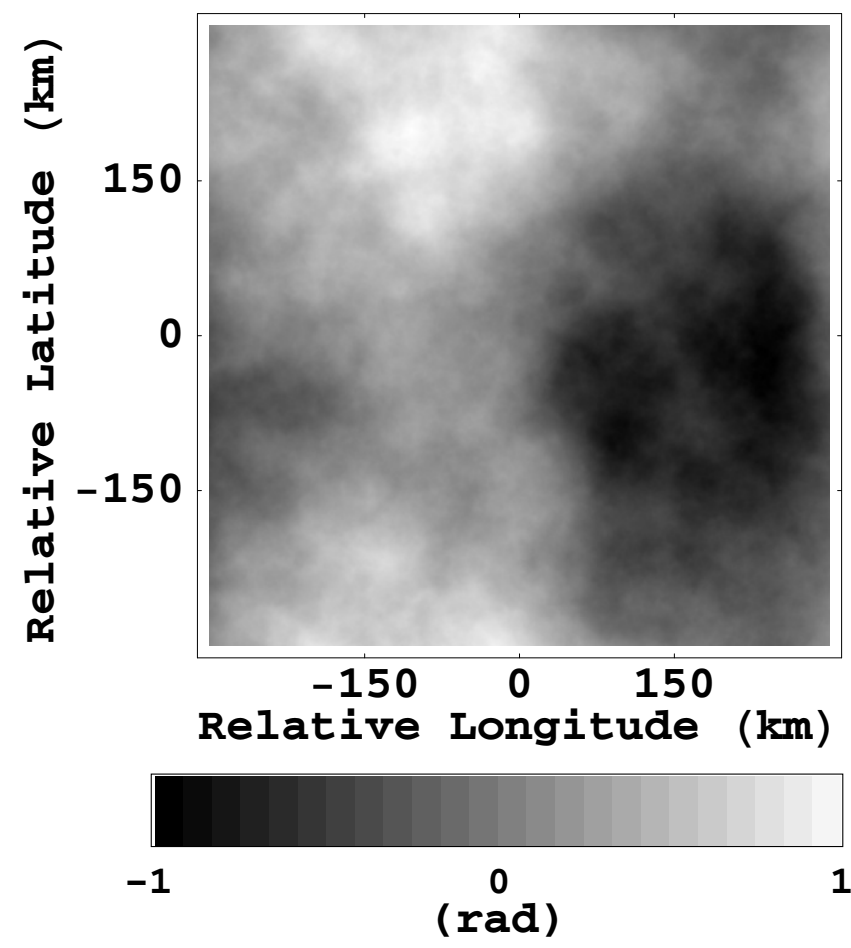

Fig. 3. Example of a turbulent phase screen with Kolmogorov statistics. The grey scale shows variations in optical-path phases, normalized between -1 and 1 radians. The final values of the phases depend (given the self-similarity of the distribution) on a global factor related to the observing frequency and the ionospheric and/or wet tropospheric conditions.

source at the same station. This numerical strategy also accelerated our simulations.

We note that we did not introduce any time evolution in the turbulent phase screens in our simulations. Any evolution of the turbulence could dramatically affect the observations if the acquisition times were longer than the coherence time of the signal, which depends on the evolution of the turbulence and the observing frequency. However, for snapshot-like observations, of the order of a fraction of a minute or so, we could consider, as a good first approximation, a constant turbulence phase screen.

Noise from the receivers was added to our model by generating a random Gaussian noise in both the real and imaginary parts of the visibilities. The mean deviation, $\sigma$, of the Gaussian noise added to the visibilities was (e.g., Thomson et al. 1991, Eq. (6.43)):

$\sigma=\frac{\sqrt{2} k}{\eta_{Q} \sqrt{\Delta v \Delta t}} \frac{1}{S_{A}}$,

where $k$ is the Boltzmann constant, $\eta_{Q}$ is the relative loss of signal caused by the correlator quantization (we used $\eta_{Q}=0.5$ ), $\Delta v$ is the observing bandwidth, $\Delta t$ is the observing time, and $S_{A}$ is the sensitivity of the stations (collecting area over system temperature, shown in Fig. 2).

\section{Estimate of dynamic range and astrometric precision}

We simulated different sets of phase-referenced observations. In all cases, the observations were snapshots of duration $t_{0}=60 \mathrm{~s}$. Longer observing times, $t$, would, in principle, increase the 
dynamic ranges and astrometric precisions shown in all the following sections as $\sqrt{t / t_{0}}$, as long as the changing atmosphere (and, therefore, the changing source positions and shapes) did not introduce important smearing effects in the images after the combination of all visibilities.

In our first series of simulations, we generated visibilities of targets with flux densities of $0.1,1$, and $10 \mu \mathrm{Jy}$ for a separation of 5 degrees between target and calibrator. A total of 1500 simulations were performed for each flux density and frequency. We used such a large separation between calibrator and target, because these simulations of phase-referenced observations can also be applied to the study of deformations of wide-field images affected by a turbulent atmosphere.

In a second series of simulations, we studied the effects of the atmosphere as a function of calibrator-to-target separation. We simulated 1500 observations at $1420 \mathrm{MHz}$ (i.e., the hydrogen line) of a source with $1 \mu \mathrm{Jy}$ for different separations from the calibrator (2-6 degrees).

In a third run of simulations, we used only one Kolmogorov screen (which can represent either ionospheric or tropospheric turbulence, depending on the observing frequency) with different Fried lengths, to study the scalability of the simulations for different source separations and/or atmospheric conditions.

In all these simulations, we added the noise from both the atmosphere and the receivers. For each simulated phase-reference image, obtained by applying uniform weighting to the visibilities, the brightness peak was found and the corresponding point source was subtracted from the visibilities. To subtract the point source, the brightness peak was shifted to the phase center of the image by multiplying the visibilities by the corresponding plane-wave factor in Fourier space. The flux density of the point source was then estimated to be the average of the real part of the resulting visibilities, and the resulting point-source model was subtracted from the data. Afterwards, a Fourier inversion of the new visibilities produced an image of the residuals, from which the root-mean-square (rms) of all the pixels was computed. On the one hand, the deviation of the brightness peak from the image center was assumed to be the astrometry error in that image. On the other hand, the source peak divided by the rms of the residuals was taken to be the dynamic range. In Fig. 4, we show the distribution of astrometric deviations and dynamic ranges for the case of a target source of $1 \mu \mathrm{Jy}$ observed at $1420 \mathrm{MHz}$ (which corresponds to an interferometric beam of $\sim 13$ mas) located at 5 degrees from the calibrator. When the distributions such as those shown in Fig. 4 were obtained, we computed the standard deviation in the astrometric corrections and the mean value of dynamic ranges for each source flux density, frequency, and separation. The first quantity was our estimate of the astrometric uncertainty, and the second quantity was an estimate of the achievable dynamic range.

\section{Results}

\subsection{Observing frequency and signal decoherence}

If the atmospheric turbulence is not taken into account and only the noise from the receivers is added to the visibilities, our simulations reproduce the dynamic ranges given by Eq. (6.53) of Thomson et al. (1991), as expected. In addition, the noise from the receivers does not introduce considerable changes in the source position of the phase-referenced images (changes of the order of $10 \mu$ as or lower).

When the turbulent ionosphere and wet troposphere are added to the simulations, the dynamic range of the images is
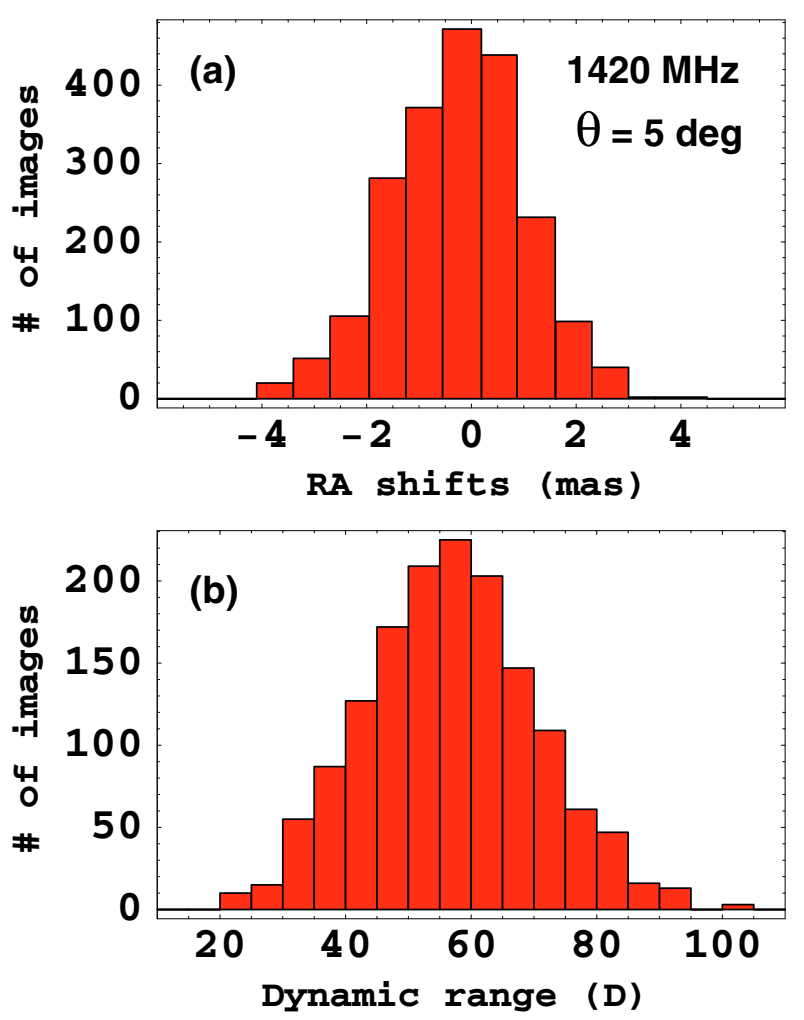

Fig. 4. Distribution of right ascension shifts a) and dynamic ranges b) of 1500 snapshot phase-referenced images, simulated at $1420 \mathrm{MHz}$, for a $1 \mu \mathrm{Jy}$ target source located at $5 \mathrm{deg}$ from its calibrator. The calibrator source is located at an hour angle of 0 and the target source is located at the zenith of the array center.

notably affected, especially at low $(<1 \mathrm{GHz})$ and high $(>10 \mathrm{GHz})$ frequencies. In Fig. 5, we show phase-referenced images of a $1 \mu \mathrm{Jy}$ source, located $5 \mathrm{deg}$. from its calibrator, observed at $0.5,5$, and $15 \mathrm{GHz}$. It can be readily seen that the inclusion of effects from the atmospheric turbulence produces an important extra noise in the images at 0.5 and $15 \mathrm{GHz}$, but not at $5 \mathrm{GHz}$.

Following the algorithm described in Sect. 4, we obtained the astrometric uncertainties and dynamic ranges shown in Fig. 6. At very low frequencies (below $\sim 500 \mathrm{MHz}$ ), the ionosphere prevents a clear and precise detection of all sources, regardless of their flux densities. At higher frequencies, the astrometric uncertainty decreases notably (mainly because of the dependence of ionospheric effects on $v^{-1}$ ) and is limited only by diffraction and sensitivity between 1 and $10 \mathrm{GHz}$ (this frequency window depends slightly on the source flux density, as can be seen in the figure). For higher frequencies, the wet troposphere begins to affect the astrometric uncertainty, which increases to around 10 mas at the highest frequencies. We find that the highest astrometric accuracy, at least for reasonably well-detected sources, is achieved for frequencies around $4 \mathrm{GHz}$. This is where the ionospheric and (wet) tropospheric components are roughly equal.

The dynamic range of the phase-referenced images is limited considerably by the atmosphere. When the atmosphere adds noise to the visibility phases, an additional rms is added to the residual images, which depends on the visibility amplitudes, thus limiting the achievable dynamic range regardless of the flux density of the source. That is, when the source flux density is higher, the noise of the image is also higher. This limitation is, of course, 


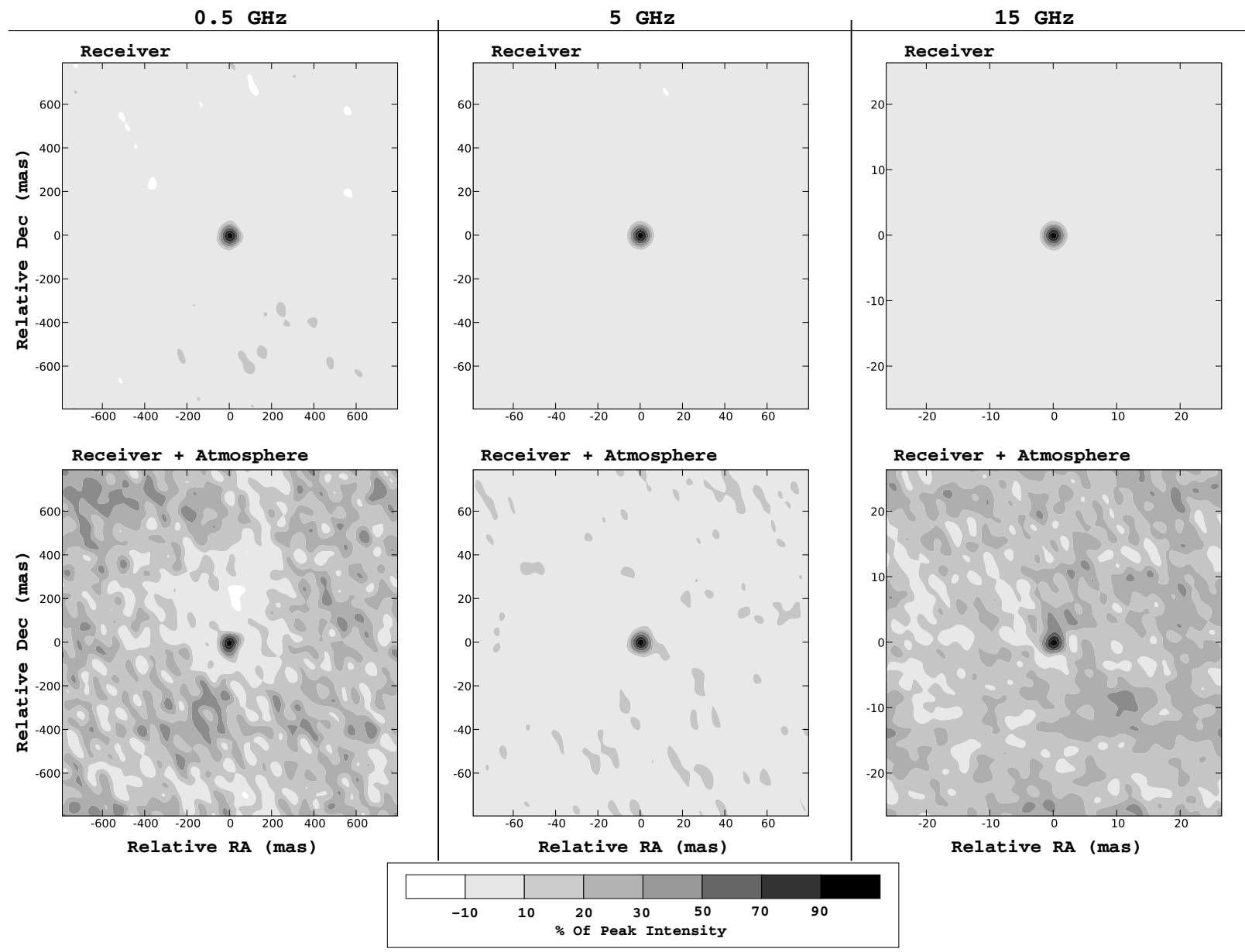

Fig. 5. Simulated phase-reference images of a $1 \mu \mathrm{Jy}$ source located at 5 deg from its calibrator. Noise from the receiving system (top) and, additionally, noise due to the turbulent atmosphere (bottom) have been added to the visibilities.

more important for the brightest sources. In our case, the brightest source has a flux density of $10 \mu \mathrm{Jy}$. For this source, the maximum dynamic range achieved is only 110 , which is $\sim 30$ times smaller than the dynamic range that would be obtained without the atmosphere. This situation can also be understood in another way. The rms of the final image can be divided into two components, which are added in quadrature. One component, $\sigma_{\text {th }}$, comes from the receiver noise and is independent of the source flux density. The other component, $\sigma_{\text {at }}$, comes from the atmospheric refraction and is equal to a percentage of the source flux density ( $\sigma_{\mathrm{at}}=K_{\mathrm{r}} S$, where $S$ is the source flux density and $K_{\mathrm{r}}$ depends on the atmospheric refraction). Hence, the dynamic range, $D$, is

$D=\frac{S}{\sqrt{\sigma_{\text {th }}^{2}+\sigma_{\text {at }}^{2}}}=\frac{S}{\sqrt{\sigma_{\text {th }}^{2}+K_{\mathrm{r}}^{2} S^{2}}} \cdot$

For high flux densities $\left(S \gg \sigma_{\text {th }}\right.$ ), the achievable dynamic range will saturate to a value that depends on the atmospheric conditions (i.e., $D \rightarrow 1 / K_{\mathrm{r}}$ ) and is independent of both the source flux density and the sensitivity of the stations. As seen in Fig. 6, despite the large difference between the simulated flux densities (1 and $10 \mu \mathrm{Jy}$ ), the dynamic range saturates to a value of $\sim 100$.

\subsection{Angular separation and signal decoherence}

The results shown in the previous subsection correspond to a separation of 5 degrees between source and calibrator. These results change when the angular separation changes. We computed astrometric uncertainties and dynamic ranges for a source with a flux densitity of $1 \mu \mathrm{Jy}$ located at 2-6 degrees from its calibrator. Noise from the atmosphere and the receivers was taken into account in these simulations. We used an observing frequency of $1420 \mathrm{MHz}$ (the hydrogen line) which is inside the frequency window where the atmospheric effects are minimised. Therefore, all the astrometric errors derived were small (of the order of a few mas), allowing us to use image sizes small enough to sample the beam with more pixels ( $\sim 30$ pixels) using a grid of $1024 \times 1024$ pixels. This fine gridding of the beam allowed us to determine the location of the image peak more accurately and, therefore, obtain a more precise estimate of the astrometric error. The results obtained are shown in Fig. 7. In that figure, we also plot two analytical (phenomenological) models for the estimate of the increase in astrometric uncertainty and the loss of dynamic range (i.e., degree of signal decoherence) as a function of angular separation. On the one hand, the phenomenological model proposed for the estimate of loss of dynamic range is

$D=\frac{D_{0}}{\sqrt{1+k^{2} D_{0}^{2} \theta^{\beta}}}$

where $D$ is the dynamic range, $\theta$ is the angular separation between target and calibrator, $D_{0}$ is the dynamic range without atmosphere (i.e., when the calibrator-to-target separation, $\theta$, tends to 0$)$, and $k$ and $\beta$ are two parameters related to the atmospheric 


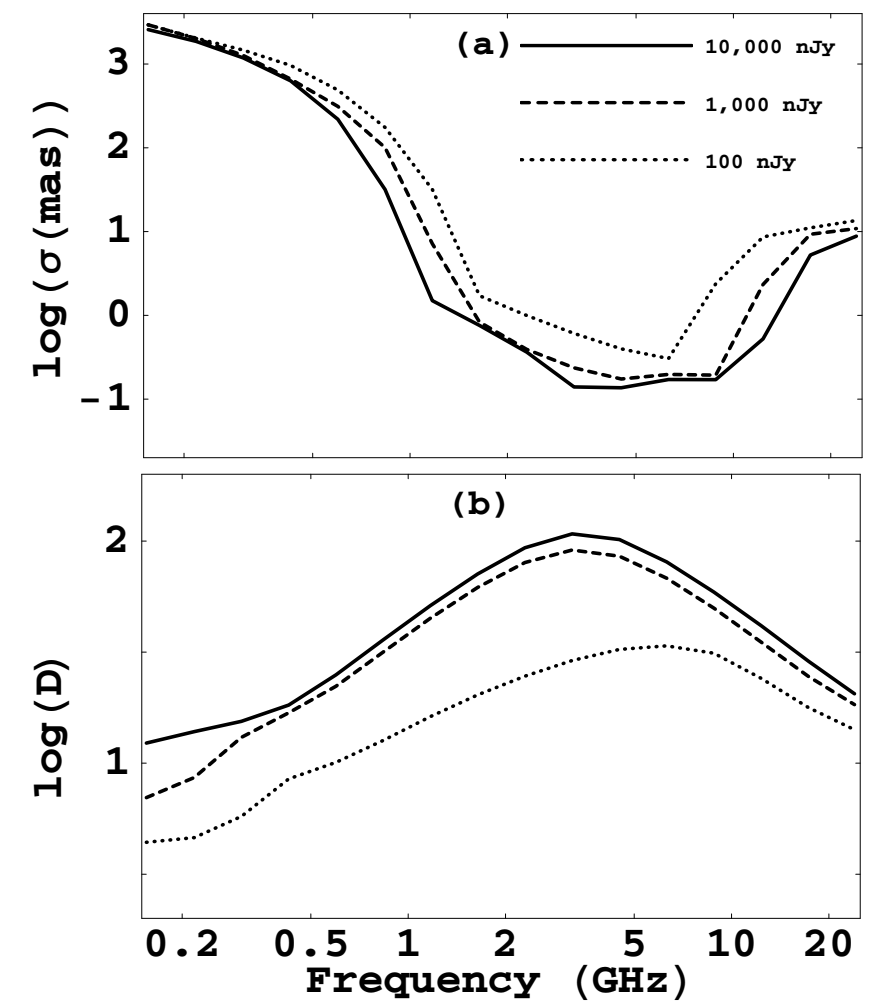

Fig. 6. Astrometric accuracy a) and dynamic range b) as a function of frequency, in the case of atmospheric turbulence and a separation of 5 degrees between calibrator and target. The calibrator source is located at an hour angle of 0 and the target source is located at the zenith of the array center. Different lines correspond to different target source flux densities (10 $\mu \mathrm{Jy}$, continuous line; $1 \mu \mathrm{Jy}$, dashed line; $0.1 \mu \mathrm{Jy}$, dotted line).

conditions, source flux density, and observing frequency. This equation is just Eq. (3), but setting $K_{\mathrm{r}}=k \theta^{\beta / 2}$.

As can be seen, this model agrees well with the simulations. We obtain $k=(2.5 \pm 0.2) \times 10^{-5} \mathrm{deg}^{-\beta}$ and $\beta=1.51 \pm 0.06$.

On the other hand, the proposed phenomenological model for the increase in the astrometric uncertainty is

$\sigma=\frac{\sigma_{0}}{D_{0}}\left(1+k^{\prime} \theta^{\beta^{\prime}}\right)$

where $\sigma$ is the astrometric uncertainty, $\sigma_{0}$ is the diffraction limit (i.e., half the size of the beam), and $k^{\prime}$ and $\beta^{\prime}$ are two parameters also related to the atmospheric conditions, source flux density, and observing frequency. We fit $k^{\prime}=0.57 \pm 0.04 \mathrm{deg}^{-\beta^{\prime}}$ and $\beta^{\prime}=1.02 \pm 0.05$. From a different approach to treat the noise from the atmosphere limited to VLBA and EVN arrays, Pradel et al. (2006) obtained a similar dependence of $\sigma$ with $\theta$.

We note that when we change $D_{0}$ by $D$ in Eq. (5), the newly fitted $k^{\prime}$ and $\beta^{\prime}$ are $1.03 \pm 0.09$ and $0.07 \pm 0.06$, respectively. This new value of $\beta^{\prime}$ is compatible with zero. In other words, the diffraction limit divided by the dynamic range of the image is an excellent estimator of the astrometric uncertainty, at least for the range of simulated calibrator-to-target separations at $1.4 \mathrm{GHz}$ (which falls within the frequency window where the atmospheric effects are minimized).

For calibrator-to-target separations larger than $\sim 6$ degrees, the situation changes. We simulated phase-referenced images for calibrator-to-target separations of up to 12 degrees, and found that the model of dynamic range given by Eq. (4) remains valid, but the astrometric uncertainty increases more rapidly,
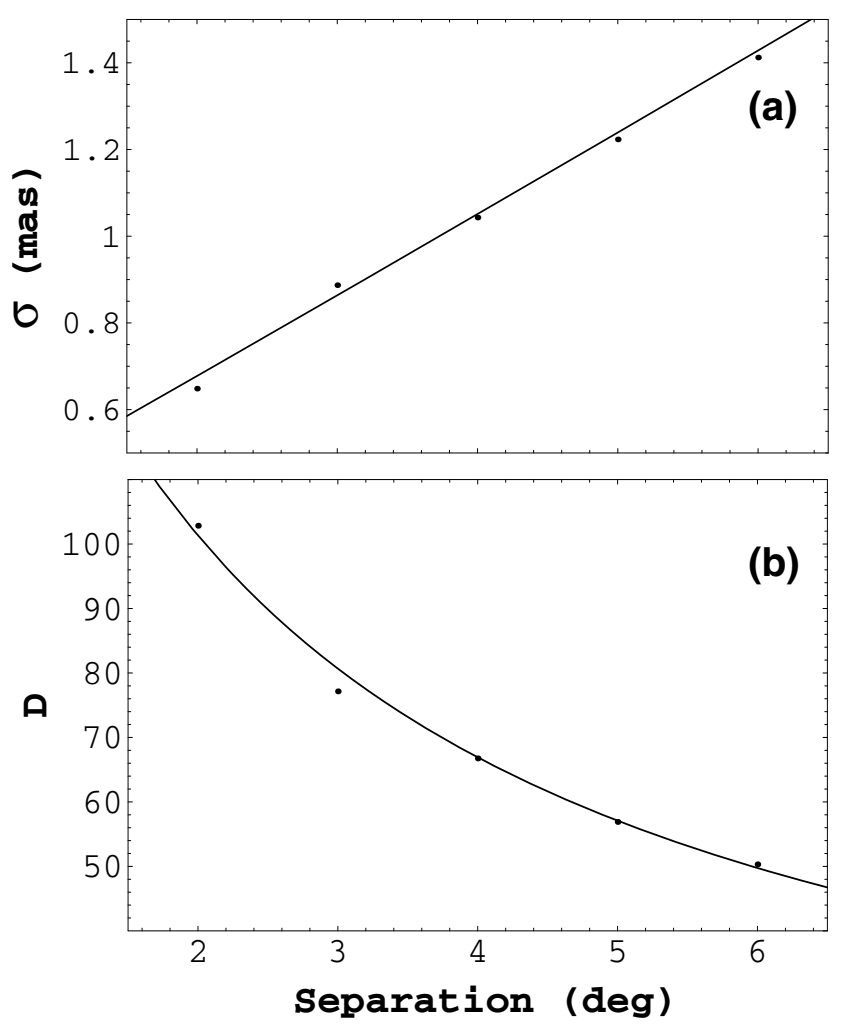

Fig. 7. Effects of target-to-calibrator separation in the quality of the phase-reference images. Dots: simulated astrometric accuracy a) and dynamic range b) for observations at $1420 \mathrm{MHz}$ and a target flux density of $1 \mu \mathrm{Jy}$. Lines: proposed phenomenological models.

with $\beta^{\prime}=2.38 \pm 0.08\left(\beta^{\prime}=1.38\right.$, if we change $D_{0}$ by $D$ in Eq. (4)). This last $\beta^{\prime}$ reproduces the astrometric uncertainties well for large source separations, but the fit is poorer for separations smaller than 5-6 degrees.

\subsection{Scalability of the results and use of turbulence models in the data calibration}

In the previous subsections, we have reported the effects of atmospheric turbulence in phase-referenced (and wide-field) interferometric images using fixed values for the Fried lengths of the Kolmogorov distributions of the ionosphere and wet troposphere. Since the Kolmogorov distribution is self-similar, the results presented can be scaled and adapted to other atmospheric conditions. These simulations can also be used to estimate the limiting dynamic range and astrometric uncertainty if an a priori model of the tropospheric and/or ionospheric turbulence is used in the imaging. In these cases, the effective Fried length, $r_{\mathrm{ef}}$, to compare to our simulations can be estimated to be

$r_{\mathrm{ef}}=r_{0}\left\langle\frac{\phi_{\mathrm{mod}}}{\left|\phi-\phi_{\mathrm{mod}}\right|}\right\rangle$

where $r_{0}$ is the Fried length of the real turbulence and the other factor is related to the fractional precision of the turbulence model, $\phi_{\text {mod }}$ is the phase computed from the turbulence model at a given point in the sky and $\phi$ is that corresponding to the real turbulence, and the brackets $<\ldots>$ represent averaging over the field of view. If the a priori model of the ionospheric electron distribution is accurate to a given precision level, the effective Fried length to use for the ionosphere will be that corresponding to the residual turbulence (i.e., the difference between the 
I. Martí-Vidal et al.: Atmospheric turbulence in interferometric images

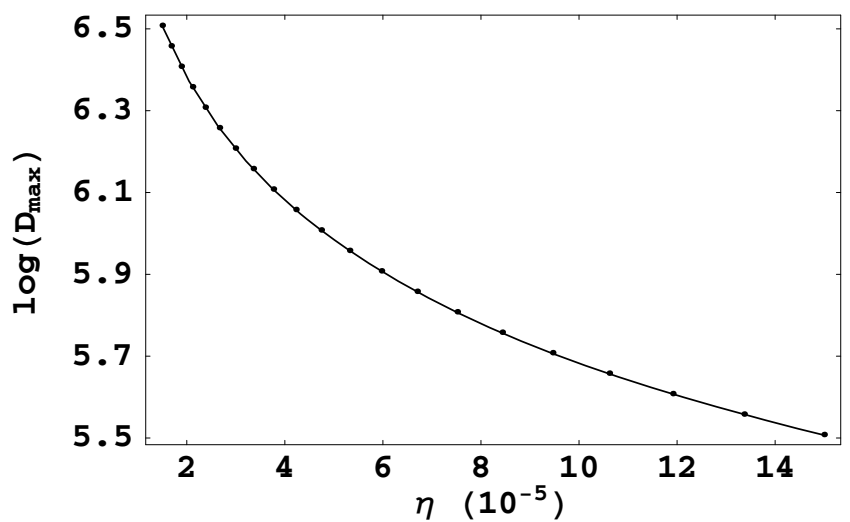

Fig. 8. Maximum dynamic range as a function of $\eta$ (see Eq. (7)) and for a 1-scan snapshot of $60 \mathrm{~s}$.

model and the real turbulence). For instance, a model of the ionospheric electron content with a $99 \%$ accuracy, has an effective Fried length of $r_{\mathrm{ef}}=100 r_{0}$; if the accuracy increases to $99.9 \%$, $r_{\text {ef }}=1000 r_{0}$. In Fig. 8, we show $D_{\max }$, the maximum dynamic range (i.e., for a source with an infinite flux density, so $\sigma_{\text {th }}=0$ in Eq. (3)) as a function of parameter $\eta$, which we define as

$\eta=\frac{h \sin \theta}{r_{\mathrm{ef}}}$

where $h$ is the height of the phase screen and $\theta$ is the calibratorto-target separation (or half the size of the wide-field image). Equation (7) can be used with Fig. 8 to compute the maximum achievable dynamic range for many different combinations of source separations, atmospheric conditions, and observing frequencies ( $r_{\mathrm{ef}} \propto v$ for the ionosphere and $r_{\mathrm{ef}} \propto v^{-1}$ for the trosposphere). We note, however, that Fig. 8 has been generated using only one Kolmogorov screen, so it is applicable to ionospheric dispersion (for low frequencies) or tropospheric dispersion (for high frequencies), but not to a situation where ionospheric and tropospheric effects are similar. In these cases, and as a first approximation, we could set

$\eta=\frac{h_{\text {ion }} \sin \theta}{\sqrt{r_{\text {ion }}^{2}+\left(\frac{h_{\text {ion }}}{h_{\text {trop }}} r_{\text {trop }}\right)^{2}}}$,

where $r_{\text {ion }}$ and $r_{\text {trop }}$ are the (effective) Fried lengths of the ionosphere and troposphere, respectively, and $h_{\text {ion }}$ and $h_{\text {trop }}$ are the heights of each phase screen. Also shown in Fig. 8 is the fitting model

$D_{\max }=D_{1} \eta^{\beta_{1}}$

where $D_{1}=48.38 \pm 0.10$ and $\beta_{1}=-1.000 \pm 0.002$. Figure 8 illustrates how difficult is to obtain a high-contrast image with a wide-angle coverage at very low (or high) frequencies. For instance, an image of $10 \times 10$ degrees with a dynamic range of $\sim 10^{6}$ at a frequency of $500 \mathrm{MHz}$ (which translates into $\eta \sim 5 \times$ $10^{-5}$ ) would require, for the ionospheric screen used in the previous subsections, a model of the ionospheric turbulence distribution with an uncertainty lower than $3 \times 10^{-5}$ (observing time of $1 \mathrm{~min}$ ). If the observing time increases to $10 \mathrm{~h}$, the minimum required uncertainty in the model of the ionosphere would increase to $7 \times 10^{-4}$, but such a high precision has to be maintained during the whole set of observations. If the dynamic range decreases to $10^{5}$, the required minimum uncertainty in the ionosphere model decreases to $7 \times 10^{-3}$ (i.e., $0.7 \%$ ) for an observing time of $10 \mathrm{~h}$.

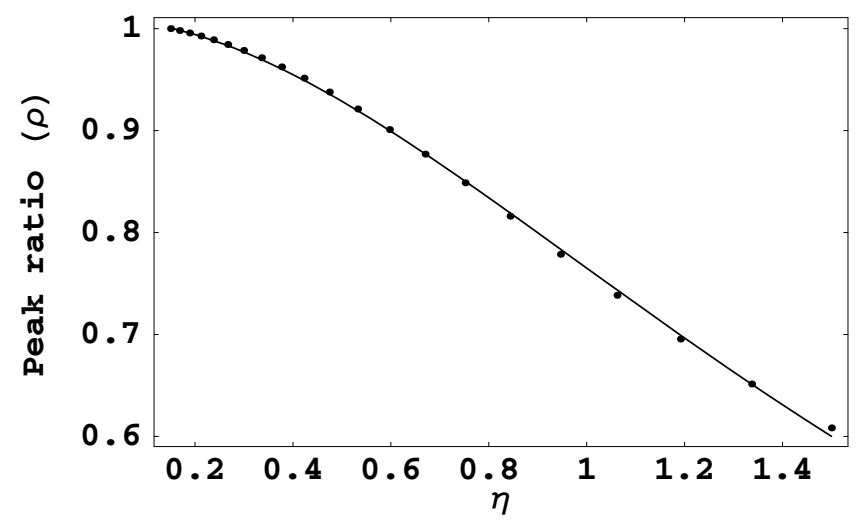

Fig. 9. Peak flux density of the phase-referenced image, relative to the real peak flux density of the source, as a function of $\eta$ (see Eq. (7)) and for a 1-scan snapshot of $60 \mathrm{~s}$.

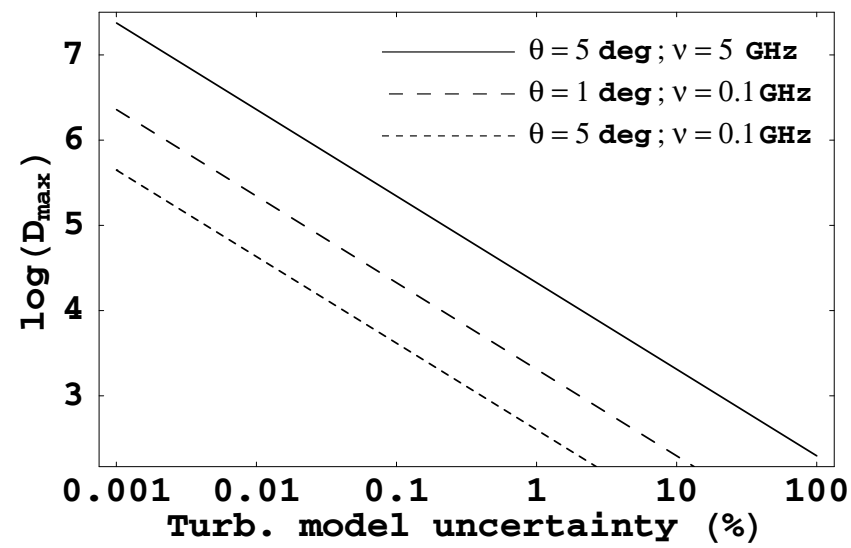

Fig. 10. Maximum dynamic range as a function of the uncertainty in the model of the atmospheric turbulence for a 1-scan snapshot of $60 \mathrm{~s}$.

For a more comprehensive representation of our results, we show in Fig. 10 how the achievable dynamic range (computed from Eq. (9)) depends on the uncertainty in the model of atmospheric turbulence used in the data calibration. We show this relationship for different observing frequencies and calibratorto-target separations. For instance, a dynamic range $10^{4}$ in observations at $100 \mathrm{MHz}$ for a calibrator-to-target separation of 1 deg (i.e., the same for a wide-field image of $2 \times 2 \mathrm{deg}$ ) would require a turbulence model with an accuracy of $\sim 99.9 \%$ for an observing time of $60 \mathrm{~s}$. This requirement would relax to an accuracy of $97-98 \%$ for an observing time of $6000 \mathrm{~s}$ (provided the dynamic range increases as the square root of the observing time).

For completeness, we also computed the loss in the recovered flux density of the source caused by the turbulent atmosphere. In Fig. 9, we show the ratio of peak flux densities of the phase-referenced images to those computed without accounting for the effects of atmospheric turbulence. For dynamic ranges of $40-50$, the loss of flux density can be as large as $25 \%$. The phenomenological model (also shown in the figure) used to fit the data is

$\rho=\frac{1}{1+D_{2} \eta^{\beta_{2}}}$

where $D_{2}=0.32 \pm 0.02$ and $\beta_{2}=1.87 \pm 0.01$. 
The applicability of Eqs. (9) and (10) is not restricted to the array used in the simulations reported here. The exponents $\beta_{1}$ and $\beta_{2}$ depend only on the structure of the atmospheric turbulence and are thus independent of the interferometer used in the observations. However, the parameters $D_{1}$ and $D_{2}$ also depend on the stations of the interferometer. Therefore, Eqs. (9) and (10) can be adapted to any other interferometer by finding the correct values of $D_{1}$ and $D_{2}$. As an example of this generalization of Eqs. (9) and (10), Martí-Vidal et al. (2010) studied the achievable dynamic range in phase-referenced observations preformed by the Very Long Baseline Array (VLBA) at $8.4 \mathrm{GHz}$ and $15 \mathrm{GHz}$. This study was based on quasi-simultaneous observations of 13 sources located at separations ranging from 1.5 to 20.5 degrees. These authors were able to model the dynamic ranges obtained in the phase-referenced images and the loss of recovered flux densities (by comparing phase-referenced images to images obtained from self-calibrated visibilities) using Eqs. (9) and (10) with values for $D_{1}$ and $D_{2}$ different to those reported here, but using the same values reported here for the exponents $\beta_{1}$ and $\beta_{2}$.

\section{Conclusions}

We have presented Monte Carlo estimates of the sensitivity and astrometric precision of an interferometric array, with a station distribution similar to that of the planned SKA, as a function of observing frequency, flux density, and source separation. These results can also be applied to other array distributions by taking into account the corresponding correction factors. Our estimates are based on simulations of snapshot phase-referenced observations, in which we take into account several effects including those of the turbulent atmosphere and the finite temperature of the receivers. We have found that the astrometric uncertainty strongly depends on the observing frequency and smoothly increases as the source separation increases. For frequencies below $\sim 1 \mathrm{GHz}$, ionospheric effects dominate and the astrometry uncertainties (when the source is detectable) can be as large as $\sim 1$ as. For frequencies between 1 and $10 \mathrm{GHz}$ (these values slightly depend on the source flux density), atmospheric effects are minimal and we roughly reach the theoretical astrometric precision of the interferometer. Above these frequencies, the wet troposphere begins to have an important effect and the astrometric uncertainty increases to $\sim 10$ mas for the highest simulated frequency $(25 \mathrm{GHz})$. The dynamic range of the images is strongly limited by atmospheric turbulence at all frequencies and for all flux densities (it can decrease, in the worst cases, by several orders of magnitude).

We have proposed analytical models for the loss of dynamic range, astrometric accuracy, and recoverable flus density as a function of distance between calibrator and target source. These expressions may also be used to estimate the deformations and local dynamic ranges of wide-field images as a function of distance to the image phase center (i.e., the point in the sky where the data correlation is centered).

Acknowledgements. We thank Ed Fomalont for his very useful comments and suggestions. I.M.V. is a fellow of the Alexander von Humboldt Foundation. This work has been supported by the European Community Framework Programme 6, Square Kilometre Array Design Studies (SKADS), contract number 011938. This work has also been partially founded by grants Prometeo 2009/104 of the GVA and AYA2009-13036-CO2-2, AYA2006-14986-CO2-01, and AYA200508561-C03 of the Spanish DGICYT.

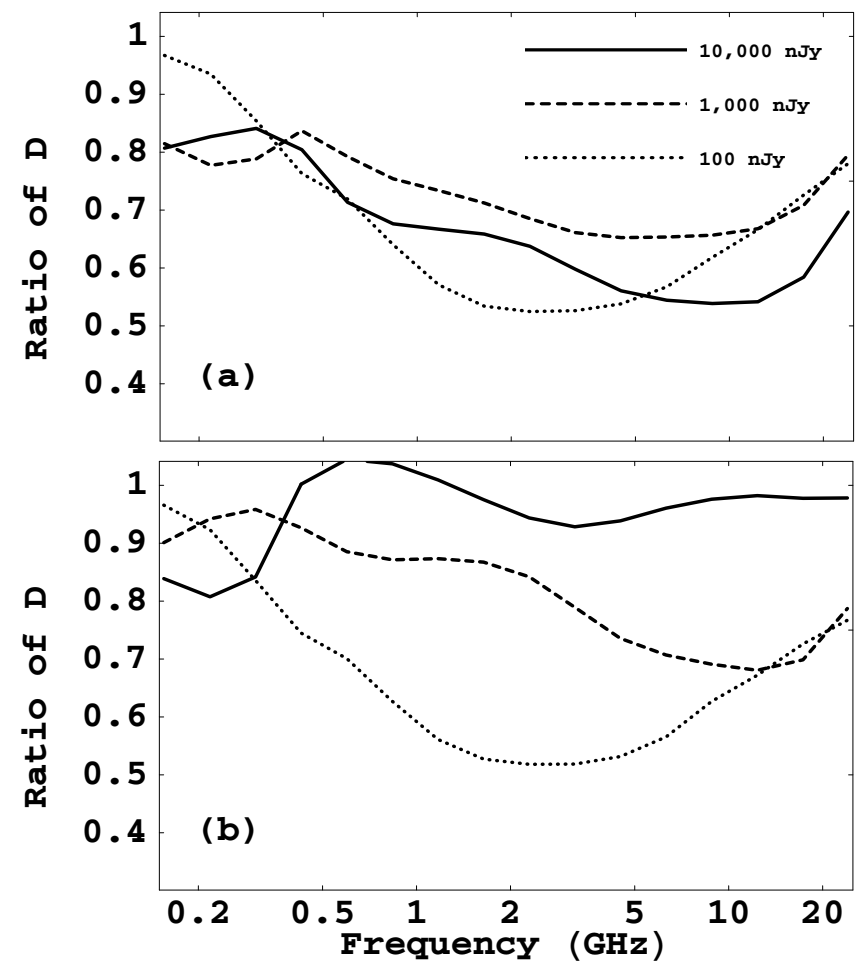

Fig. A.1. Ratios of dynamic ranges obtained with our original array and those obtained with a subarray of 100 stations a) and an array with half the sensitivity of the original array b). Different lines correspond to different target source flux densities (10 $\mu \mathrm{Jy}$, continuous line; $1 \mu \mathrm{Jy}$ dashed line; $0.1 \mu \mathrm{Jy}$, dotted line).

\section{Appendix A: Complementary simulations: different number of stations and array sensitivities}

Our simulations are based on a given realization of the SKA. However, the main structure of the array distribution used in our simulations is not exclusive to the SKA. Other interferometric arrays, such as ALMA or LOFAR, are being built with similar station distributions, consisting of a compact core and several extensions along the shape of spiral arms. Hence, our study can be extended to those arrays by taking into account the difference between the number of stations and the station sensitivities.

The amount of noise added to the data is proportional to the number of stations, since each station receives the signal through the turbulent atmosphere. However, in cases of clear source detections $(D>20-30)$, the dynamic range does not depend (or the dependence is weak) on the thermal noise of the receivers (see Sect. 5.1). Therefore, to estimate the achievable dynamic range for an array with a different number of stations, the results shown in Fig. 6 should be divided by $N / N^{\prime}$, where $N$ is the number of stations used in our simulations $(N=200)$ and $N^{\prime}$ is the number of stations of the other array. This is true for detections with a relatively large dynamic range $(D>20-30)$. For weak sources, the noise from the receivers may also contribute to the rms of the residual images, so the factor to apply in these cases should be $\left(N \sigma^{\prime}\right) /\left(N^{\prime} \sigma\right)$, where $\sigma$ is the thermal noise of the stations used in our simulations and $\sigma^{\prime}$ is that of the other array.

We repeated the simulations described in Sect. 5.1 using different arrays to compare the results with those obtained with the original array. On the one hand, we created a smaller array by subtracting 100 stations (those marked with empty squares in 
Fig. 1) from the original array. On the other hand, we created another array with all the 200 stations, but decreasing their sensitivity by a factor of 2 . We show the results obtained in Fig. A.1. Special care must be taken interpretating these figures, given that the computed ratios of dynamic ranges are only meaningful when the detections of the sources are clear (i.e., when no spurious noise peaks appear stronger than the source). This is true for $D>20-30$, which corresponds approximately to frequencies between 1 and $10 \mathrm{GHz}$ (although it depends slightly on the source flux density, see Fig. 6).

By taking these considerations into account, we find that the ratio of dynamic ranges for an array with 100 stations falls between 0.7 and 0.5 compared to the array with 200 stations. The expected value is 0.5 ( since $N^{\prime}=0.5 N$ and $\sigma^{\prime}=\sigma$ ). Other factors, such as the different coverages of Fourier space by both arrays, may affect the dynamic range of the images, thus increasing the ratio in some cases. For an array with lower station sensitivities (but the same number of stations), the ratio of dynamic ranges falls between 0.75 and 1 for the strongest sources (as expected, since $N^{\prime}=N$ and the noise from the receivers is much smaller than the noise from the atmosphere), but is close to 0.5 for the weakest source (also as expected, since the thermal noise from the receivers begins to dominate in this case).

\section{References}

Eckers, R. D. 1999, in Synthesis Imaging in Radio Astronomy II, ed. Taylor, Carilli, \& Perley, ASP Conf. Ser., 180

Jones, D. L. 2004, SKA Memo, 45

Martí-Vidal, I., Guirado, J. C., Jiménez-Monferrer, S., \& Marcaide, J. M. 2009, SKA Memo, 112

Martí-Vidal, I., Ros, E., Pérez-Torres, M. A., et al. 2010, A\&A, 515, A53

Pradel, N., Charlot, P., \& Lestrade, J.-F. 2006, A\&A, 452, 1099

Readhead, A. C. S., \& Wilkinson, P. N. 1978, ApJ, 223, 25

Schilizzi, R. T., Alexander, P., Cordes, J. M., et al. 2007, SKA Memo, 100

Thomson, A. R., Moran, J. M., \& Swenson, G. W. 1991, Interferometry and Synthesis in Radio Astronomy (Florida: Krieger Publ. Corp.)

van der Tol, S., Jeffs, B. D., \& van der Veen, A. J. 2007, in IEEE Tr. Signal Processing

Vir Lal, D., Lobanov, A. P., \& Jiménez-Monferrer, S. 2009, SKA Memo, submitted 